\title{
Nurses' Perceived Barriers to and Facilitators of Research Utilization in Mainland China: A Cross-Sectional Survey
}

\author{
Wai-Tong Chien ${ }^{*}, 1$, Qin Bai ${ }^{2}$, Wai-Kit Wong ${ }^{1}$, Huizhen Wang ${ }^{3}$ and Xueqin $\mathrm{Lu}^{2}$ \\ ${ }^{1}$ School of Nursing, Faculty of Health and Social Sciences, The Hong Kong Polytechnic University, Hong Kong \\ ${ }^{2}$ Nursing College of Henan University, Kaifeng, Henan Province, P.R. China \\ ${ }^{3}$ Nursing College of Southern Medical University, Guangzhou, Guangdong Province, P.R. China
}

\begin{abstract}
Despite the drive towards evidence-based practice, the extent to which research evidence is being implemented in nursing practice is unclear, particularly in developing countries. This study was to assess the levels of perceived barriers to and facilitators of research utilization in practice among Chinese nurses and inter-relationships between these barriers and facilitators and their socio-demographic characteristics. A cross-sectional, descriptive survey was conducted in 2011 with 743 registered nurses randomly selected from four general hospitals in China. They completed the Barriers to Research Utilization and Facilitators of Research Utilization scales. Correlation tests were used to test the relationships between the nurses' perceived barriers and facilitators, their demographic characteristics and research training and involvement. The Chinese nurses' level of perceived barriers was moderate on average and lower than that in previous research. Among the 10 top-ranked items, six were from the subscale 'Organizational Characteristics'. Their perceived barriers were correlated positively with age and post-registration experience and negatively with research training undertaken. Junior diplomatic nurses reported a significantly higher degree of barriers than those senior ones with postgraduate education. Higher and more diverse barriers to research utilization in practice are perceived by Chinese nurses than those in Western countries and they are associated with a few socio-demographic factors. Future research on these barriers/facilitators and their relationships with occupational and socio-cultural factors in Chinese and other Asian nurses is recommended.
\end{abstract}

Keywords: Cross-sectional survey, perceived barriers, perceived facilitators, research utilization, registered nurses, Chinese.

\section{INTRODUCTION}

Due to the increasing societal expectation of high quality health care, nursing as one of the practice disciplines should meet the demand for evidence-based practice that are advocated by both service users and health care organizations. Nurses are expected to establish and make use of the best research evidence to enhance the clinical effectiveness of patient care and improve patients' health outcomes. The application of research evidence to everyday practice may not only elevate the quality and standards of nursing care [1], but also enhance personal and professional growth for nurses themselves [2]. It is no longer acceptable for nurses to deliver nursing care on a ritualistic and traditional basis [3]. The development and continuation of professional status in nursing should include an increased awareness of the need for a research-based practice and scientific knowledge generated from it to guide and improve clinical practice.

Although similar research has been conducted in several countries, there are differences reported between nurses in different Western and Asian countries in relation to great

*Address correspondence to this author at the School of Nursing, Faculty of Health and Social Sciences, The Hong Kong Polytechnic University, Hong Kong, SAR, P.R. China; Tel: (852)2766 5648; Fax: (852)2364 9663;

E-mail: wai.tong.chien@polyu.edu.hk variations between the cultures and organizational contexts [4-6]. In addition, much remains not to be understood about research utilization for improved nursing practice, especially in newly-industrialized, developing countries where nurses in the past have been little involved with research $[7,8]$. The factors facilitating and/or inhibiting research utilization in practice have not yet been identified in the health care system of mainland China, particularly in the discipline of nursing.

\section{LITERATURE REVIEW}

Although nurses may have positive attitudes and values overall towards research or research-based practice [9-11], the transfer of research evidence into nursing practices and education remains slow, and is sometimes unsuccessful [12]. To understand this slow development of research-based practice in nursing, it is important to examine the possible factors influencing nurses' successful transfer and implementation of research evidence into their practice.

One of the most important areas of research on these factors is an understanding of the involved nurses themselves and of their organizational context and culture, in terms of both their perceived barriers to and facilitators of research utilization in their practice settings. Several studies in developed Western and Asian countries have found a few common important perceived barriers and facilitators among nurses. However, major differences on both of these barriers 
and facilitators have also been identified across countries due to the great variations between the cultures and organizational contexts $[6,8]$.

Squires et al. [13] conducted a systematic review on research evidence utilization, indicating that individual factors of health professionals such as personal beliefs and attitudes, involvement in research activities, and a few selected socio-demographic and professional characteristics (e.g., education level, rank position and age) might affect their perceptions of the value of research evidence applied to their practice. Being similar among developed Western countries, a lack of understanding about research methods, statistical knowledge and critical appraisals of research findings have been cited as the most common personal barriers to research utilization among health professionals, including nurses [4]. Continuing education to develop nurses' knowledge and skills in understanding about research design, appraising research literature and participating in research are recommended to improve the extent of research utilization among nurses [14].

In another literature review, Hutchinson and Johnston [15] indicated that the most reported worldwide barriers perceived by nurses included mainly a few organizational or work setting factors, which were a lack of autonomy or authority to change practice, inadequate facilities for research, limited cooperation and support from the management level and physicians, and a lack of time to implement new ideas and read research. While initiating changes and updates in clinical practice have been required by the management level as an integral part of nursing [16], the limited organizational support perceived by nurses as the most common barriers to research utilization in many recent studies compromises the development of evidence-based nursing practice $[4,14,17]$. This makes such important changes in practice a challenge to all nurses. In addition, due to the rapid development of the organizational structure and system of health care in some developing countries such as mainland China, nurses' perceived barriers concerning organizational support and their characteristics and competence could vary from the recent findings on research utilization in Western countries and thus should be investigated in order to better understand nurses' current perceived barriers to research utilization in those countries.

Research factors such as quality and communication of research findings have been frequently rated as the second most important set of nurses' perceived barriers in recent research (e.g., Chau et al. [18], LaPierre et al. [19] and Mckenna et al. [20]). Research reports with too brief a presentation of research evidence or unclear implications for nursing practice could be seen as a major barrier to evidencebased practice in primary care nurses [21]; conflicting results and perceived gaps between theory, research evidence and practice are considered to be the most important barriers by community nurses [20]. Therefore, the major challenge for applying research evidence into practice can be closely related to the nurses' perceptions of the extent to which the research findings are directly relevant and useful to the practice and management issues in their settings. This shows that the research problems and/or objectives should consider nurses' current concerns and needs for practice.
Recently, a few studies of nurses' perceived barriers to research utilization have been conducted in Western countries, particularly in Europe (e.g., Bryar et al. [17] in the United Kingdom; Oranta, Routasalo and Hupli [22] in Finland; and Parahoo [23] in Northern Ireland). Funk et al. [24] developed a self-report questionnaire entitled the Barriers to Research Utilization (BARRIERS) scale, which has been frequently used in recent studies, to assess nurses' perceptions in regard to barriers to research utilization in their practice. The BARRIERS scale adopted Rogers' [25] model of 'diffusion of innovations', a theoretical framework to describe the process of communication through channels within a social network, of an idea, practice or actions over time. The 29-item BARRIERS scale has been validated among diverse groups of nurse practitioners and managers in different countries, indicating satisfactory reliability and validity [15]. Funk et al. [24] also undertook an exploratory factor analysis to elicit a four-factor solution that was closely related to Rogers' [25] model, including characteristics of nurses, qualities of research, presentation of research findings, and organizational characteristics. However, although further factor analyses of the scale in recent studies resulted in minor revisions on its factor structure, most studies have found that the four-factor solution accounts for only $45 \%$ of the total variance.

A recent study conducted by Hutchinson and Johnston [4] with 960 registered nurses in Melbourne, Australia identified the four-factor solution as the most appropriate model arising from Principal Components Analysis of the 29 items. The factor groupings were similar to those of the original authors [24]; however, a few items were grouped into factors different from those of the original authors, for example, 'The nurse is unwilling to change or try new ideas' and 'The research is not relevant to the nurses' practice' were moved from 'Characteristics of Nurses' to 'Organizational Characteristics' and from 'Presentation of Research Findings' to 'Qualities of Research', respectively. An eight-item Facilitators of Research Utilization (FRU) scale was also developed by Hutchinson and Johnston [4] to assess nurses' opinions about the supporting factors of research utilization in their settings and its reliability and validity was then reported in Chau et al. [18] to be very satisfactory (i.e., the Cronbach's alpha was 0.84 and the content validity as rated by an expert panel of health professionals was high).

Most recent research findings using the BARRIERS scale identified a few common perceived barriers, including inadequate time to read, interpret and implement new ideas of research findings [5,18]; knowledge deficits in accessing, understanding and evaluating research findings $[8,15,26]$; relevant research findings not being accessible in the workplace $[6,18]$; and lack of autonomy and authority to change practice [6,7]. Several aspects of nursing culture such as ritualistic care and lack of incentives to develop researchbased practice have also been found to contribute to nurses' perceived barriers to research utilization [27]. Hutchinson and Johnston [4] also suggested that the influence of health care system, culture and organizational characteristics may also be distinctive across countries, particularly with possible great differences between the well-established and open system in many Western countries and the traditional, 
hierarchical top-down structure in many Asian countries $[18,28]$.

As a matter of fact, there is a lack of research on these perceived barriers and facilitators from the nurses' point of view in fast-developing countries such as mainland China and India. Therefore, this study aimed to investigate the perceived barriers to and facilitators of research utilization among a large group of hospital nurses in large cities of China, which has recently demonstrated consistent, rapid development of its health care system and nursing services [29].

\section{METHODS}

\section{Aim/Objectives}

The objectives of the study were:

1. To assess the overall levels of perceived barriers to and facilitators of research utilization in nursing practice among Chinese registered nurses in four general hospitals of mainland China;

2. To identify the top and/or bottom ranked barriers to and facilitators of research utilization among these Chinese nurses; and

3. To examine the relationships between the perceived barriers, perceived facilitators, and sociodemographic and professional characteristics of these Chinese nurses.

\section{Design}

This was a cross-sectional, descriptive correlational study conducted between March and June 2011 to elicit nurses' perceptions of the barriers to and facilitators of research utilization in their practice. The self-reported questionnaire, consisting of the 29-item BARRIERS and 8-item FRU scale, was a simple and direct measure to obtain registered nurses' perceptions of the obstacles to and facilitators of researchbased practice. This study design, and the valid instruments used, would also allow the researchers to identify, compare and explain the nurses' perceived barriers and facilitators in relation to the subgroup characteristics of the nurses [30].

\section{Sample}

Participants comprised 800 Chinese registered nurses from four regional general hospitals in the Henan and Guangdong provinces of mainland China. They were randomly selected from the staff lists of the four hospitals using computer-generated random numbers. The hospitals were government-funded metropolitan hospitals (in Zhengzhou and Kaifeng of Henan and Guangzhou and Shenzhen of Guangdong), serving a total population of about two million people. Each of these four hospitals consisted of more than 1,000 beds and a comprehensive coverage of acute, palliative and outpatient care departments and pathological and diagnostic units. In each of the four hospitals under study, there were a small library with a few computers containing two to three databases (e.g., Medline and CINAHL) for literature search and a few sets of medical and nursing journals (e.g., British Medical Journal and Journal of Clinical Nursing). They did not have any research centre, committee, theme group, or ethics committee. Annually, a few nurse managers and senior registered nurses could be nominated and sponsored to attend one international nursing conference, and/or one short-term course relevant to nursing research or evidence-based practice. In addition, two of the four hospitals also organized a few bi-monthly seminars regarding clinical research or evidence-based practice for all hospital staff to share about the findings of research conducted in their clinical departments.

There were a total of approximately 1,400 registered general nurses in the hospitals at subject recruitment, representing about $2 \%$ of the total registered nurses in China. According to Wang et al. [31], this sample size allowed for a $5 \%$ sampling error with 95\% confidence level, taking account of a non-response rate of up to $20 \%$. Nevertheless, a higher response rate would be desirable to increase the internal validity of the study.

Inclusion criteria for participants included those who:

a. were registered general nurses who had been graduated for more than three months;

b. were working full-time in one of the hospitals under study; and

c. agreed to participate voluntarily in the study.

Those who had been absent from the hospital or on long leave (e.g., maternity leave) over the previous three months at the time of participant recruitment were excluded.

\section{Instruments}

The self-administered questionnaire consisted of three sections and required about 15 minutes to complete. The first section was the demographic data sheet, mainly consisting of nurses' age, gender, marital status, education level, years of post-registration experience, rank position, specialty area of nursing, training in basic nursing research (undertaken in the past three years), and involvement in clinical research.

The second part was the self-reported 29-item Barriers to Research Utilization (BARRIERS) scale developed by Funk et al. [24] and translated into Chinese language by Chau et al. [18] for Hong Kong Chinese nurses. The respondents were asked to rate each item as to what they perceived to be the barriers to their use of research or its findings in practice, on a 5 -point Likert-type scale, from $1=$ 'to no extent' to $5=$ 'to a great extent'. The translated scale consisted of four subscales: Nurse Characteristics (originally referred to 'The Nurse'; 9 items), Quality of Research ('The Research'; 6 items), and Organizational Characteristics ('The Setting'; 8 items), and Presentation and Accessibility of Research ('Communication and Presentation of Research'; 6 items). The Chinese version has indicated high internal consistency (i.e., Cronbach's alphas ranged from 0.71-0.93) and satisfactory test-retest reliability (Pearson's $r=0.68-0.83$ ), as well as satisfactory content and construct validity [6]. In addition, the questionnaire had a question to elicit the respondents' three greatest perceived barriers to research utilization.

The third section was the 8-item Facilitators of Research Utilization (FRU) scale developed by Hutchinson and Johnston [4]. It was also translated into Chinese by Chau et al. [18], indicating high content validity and internal consistency (i.e., the Cronbach's alpha was 0.84). 
Respondents also rated their answers on the same 5-point Likert-type scale as used in the BARRIERS scale. Both Chinese versions had been re-formatted into simplified Chinese language (official written language) without changing any wording or meaning, and reviewed by an expert panel in mainland China, consisting of two nurse researcher, one nurse educator, and two senior registered nurses, on its appropriateness and relevance (two sets of ratings, ranging from 1 - not appropriate/relevant to 4 - very appropriate/relevant) to their clinical settings. All of the items on the barriers and facilitators scale were rated as content and linguistic relevant and appropriate (i.e., 3 or 4), except three words/terms (i.e., "research articles", "generalizable" and "clinically focused") needing minor amendments.

\section{Ethical Considerations}

Ethical approvals and permission to access the study venues were gained from the ethics committees of the four hospitals under study and the Human Subjects Research Ethics Committee of The Hong Kong Polytechnic University. Permission to use the BARRIERS scale was granted by the original author. A cover letter with full information about the study was attached with the anonymous questionnaire and return of the completed questionnaires was taken to imply consent to participate in the study. The questionnaire and personal data were kept confidential and only accessed by the researchers. The nurses were assured that their participation in this study was on a voluntary basis.

\section{Data Collection}

Registered nurses who were randomly selected from the staff lists of the four hospitals were invited to complete the questionnaire, consisting of the self-reported BARRIERS and FRU scales and the demographic data sheet. To enhance the response rate, the questionnaires attached to the cover letter were hand delivered to the selected nurses by one of the researchers. A self-addressed envelope was also provided for the nurses to deposit the completed questionnaires into a collection box on their ward. The contact telephone number of one of the researchers was included in the letter in case the nurses needed to ask questions about the study.

\section{Data Analysis}

Data from the study questionnaires were numerically coded for quantitative analysis. Descriptive and inferential statistics were calculated using the PASW (formerly SPSS), version 17. Descriptive statistics such as frequency, mean and standard deviation were used to describe the sociodemographic characteristics of the nurse respondents and to summarize the BARRIERS and FRU scores. A list was made of top- and bottom-ranked barriers rated four or five by most of the respondents, as well as of all eight items related to facilitators. Pearson's and Spearman's correlation tests were used to examine the inter-relationships between the respondents' characteristics (in ordinal or interval levels of measurement, respectively) such as their age, education level and years of post-registration experiences and their perceived barriers to and facilitators of research utilization. Bi-serial correlation test was used to test their inter-relationships between the mean scores of the nurses' barrier and facilitator scores and their training and involvement in research. Independent t-test or one-way analysis of variance (ANOVA) test was used to compare nurses' BARRIERS and FRU mean scores between subgroups of selected characteristics, including their education level, postregistration nursing experience, specialty area, and rank position [30]. Tests of significance were two-tailed, with values of $\mathrm{p}<0.05$ considered significant.

\section{RESULTS}

\section{Descriptions of the Participants}

There were 743 nurses who ultimately completed and returned the questionnaires, yielding a response rate of $92.8 \%$. The demographic characteristics of the respondents are summarized in Table 1 . All respondents were female and their mean age was 30.19 ( $\mathrm{SD}=7.23$ ), ranging from 21 to 49 years old. About two-thirds $(60.8 \%)$ of them were married and less than half $(43.6 \%)$ had obtained a bachelor degree or above in nursing. Over two-thirds of them were working in medical or gerontological $(42.0 \%)$ and surgical, orthopedic or operating theatre $(25.6 \%)$ units. There was a wide range of post-registration nursing experience among the respondents, mainly from one to 12 years $(58.4 \%$ with $1-10$ years and $20.7 \%$ with $11-13$ years). About half of them $(50.2 \%)$ had undertaken their basic research courses during basic nursing or in-service, post-registration education over the past three years. Nevertheless, only $15.6 \%$ of the nurses had participated in clinical research since nursing registration. There were no significant differences in all of these characteristics between the study participants and those from the 2009 Hospital Statistics of Registered Nurses $[32,33]$ for the two provinces in which the study hospitals were situated, using a Goodness-of-Fit Chi-square test.

\section{Questionnaire Results}

\section{Nurses' Perceived Barriers to Research Utilization}

The means (and 95\% confidence intervals), standard deviations and score ranges and Cronbach's alphas of the BARRIERS scale and its subscales are summarized in Table 2. The majority of respondents rated approximately 20 $(69.0 \%)$ of the 29 barrier items as moderate or great barriers. Only $5.4 \%$ to $9.5 \%$ of the nurses rated 'No opinion' on the 29 items of the BARRIERS scale. The mean total score of the BARRIERS scale $(89.32, \mathrm{SD}=19.62 ; 95 \% \mathrm{CI}=87.91$ 90.73) was at moderate level (i.e., in the middle of the possible score range from 29 to 145). Among the four subscales of perceived barriers to research utilization, the mean score of the 'Organizational Characteristics' (mean=26.09, $\mathrm{SD}=6.25$; possible score range from 8 to 40 ) was the highest. This indicates that the Chinese nurses might perceive organizational factors to be the most important sources of barriers to research utilization in different settings. The Cornbach's alphas of the overall BARRIERS scale and subscales ranged from 0.87 to 0.92 .

\section{The Most and Least Important Perceived Barriers}

The 10 top- and five bottom-rated barriers perceived by the Chinese nurses in terms of importance, together with their means, standard deviations, numerical ranking, and 
Table 1. Socio-Demographic Characteristics of Chinese Nurses $(\mathbf{N}=743)$

\begin{tabular}{|l|l|}
\hline Characteristics & \multicolumn{1}{|c|}{$\begin{array}{c}(\%) \\
\end{array}$} \\
\hline \hline Age in years (mean, SD) & $(30.19,7.23)$ \\
$20-25$ & $112(15.07)$ \\
$26-30$ & $231(31.90)$ \\
$31-35$ & $278(37.42)$ \\
$36-40$ & $78(10.49)$ \\
Over 40 & $44(5.92)$ \\
\hline Educational level & \\
Certificate (technical school) & $22(2.96)$ \\
Diploma (college or high school) & $397(53.43)$ \\
Undergraduate degree & $306(41.18)$ \\
Master's degree or above & $18(2.42)$ \\
\hline Marital status & \\
Single & $208(28.00)$ \\
Divorced & $83(11.17)$ \\
Married & $452(60.83)$ \\
\hline Specialty nursing area & \\
Medical/geronotological & $312(41.99)$ \\
Surgical/orthopaedic/operating theatre & $190(25.57)$ \\
ICU/critical care & $98(13.19)$ \\
Obstetric/gynaecologic & $76(10.23)$ \\
Others (e.g., A\&E and day care centre) & $67(9.02)$ \\
\hline Post-registration nursing experience & $63(8.48)$ \\
Less than one year & $287(38.63)$ \\
1-5 years & $147(19.78)$ \\
6-10 years & $154(20.73)$ \\
11-13 years & $92(12.38)$ \\
More than 13 years & \\
\hline Nursing research course undertaken in previous three years \\
Yes & $370(40.20)$ \\
No & \\
\hline Participation in clinical research & \\
Yes & \\
No & \\
\hline
\end{tabular}

subscales are presented in Table 3 . The item mean scores of the perceived barriers ranged from $2.81(\mathrm{SD}=1.03)$ to 4.10 $(\mathrm{SD}=1.20)$, with possible item scores ranging from 1 to 5 . Of the 10 top-ranked items, six were from the subscale
'Organizational Characteristics' and three were from the subscale 'Nurses Characteristics'. The six top ranked barriers from the subscale 'Organizational Characteristics' were mainly related to limitations of resources, facilities and nursing manpower in the nurses' workplaces or organizations, for example, 'There is insufficient time on the job to implement new ideas' (i.e., the top barrier) and 'The facilities are inadequate for implementation' (i.e., the third barrier). In addition, three of the professional characteristics of the nurses themselves were also perceived as major barriers to research utilization in their practice, for example, 'The nurse does not see the value of research for practice nurses' (i.e., the second one) and 'The nurse is unaware of the research' (i.e., the fifth one).

The five least rated barriers included three items from the 'Nurses' Characteristics' and two from 'Quality of Research', with mean scores ranging from 2.81 ( $\mathrm{SD}=1.03$ ) to $2.93(\mathrm{SD}=1.13)$. The two least rated items were: 'The nurse is unwilling to change/try new ideas' (i.e., the $28^{\text {th }}$ barrier) and 'The nurse sees little benefit for self' (i.e., the $29^{\text {th }}$ one).

Results from the question that asked the Chinese nurses to cite the three greatest barriers to research utilization indicated very similar results to the above item mean scores. The five most frequently cited greatest barriers to research utilization included two of the above-mentioned top-ranked items from the subscale 'Organizational Characteristics', namely 'There is insufficient time on the job to implement new ideas' (36.3\%) and 'The nurse does not have time to read research' (34.2\%). Another two of the greatest barriers were from the subscale 'Nurses' Characteristics', namely, 'The nurse is unwilling to change/ try new ideas' (33.1\%) and 'The nurse does not see the value of research for practice' $(29.8 \%)$. The remaining one was from the subscale 'Presentation and Accessibility of Research', namely, 'Implications for practice are not made clear' (28.7\%).

\section{Levels of Facilitators of Research Utilization Obtained}

The item means and standard deviations of the 8-item FRU scale and the ranking in descending order of the item means are summarized in Table 4 . Only $5 \%$ to $8 \%$ of the nurses gave 'No opinion' on the 8 items of the scale. The item mean values ranged from $3.52(\mathrm{SD}=1.25)$ to 3.92 $(\mathrm{SD}=1.14)$, with a possible range from 1 to 5 . The three top perceived facilitators included: 'Advanced education to increase your research knowledge base' (mean $=3.92$, $\mathrm{SD}=1.14)$, 'Improving availability and accessibility of research reports' (mean $=3.89, \mathrm{SD}=1.09)$ and 'Enhancing managerial support and encouragement of research

Table 2. Means and Standard Deviations of Item Scores of the BARRIERS Scale and its Subscales $(\mathrm{N}=743)$

\begin{tabular}{|lcccc|}
\hline \multicolumn{1}{|c}{ Scale or Subscales } & Actual Range & Mean (SD) & 95\% CI & Cronbach's Alpha \\
\hline \hline${\text { BARRIERS total scale }(29-145)^{\mathrm{a}}}$ & $35-145$ & $89.32(19.62)$ & $87.91-90.73$ & 0.90 \\
Organisational characteristics $(8-40)$ & $10-40$ & $26.09(6.25)$ & $25.64-26.53$ & 0.87 \\
Nurse characteristics $(9-45)$ & $9-40$ & $24.61(6.42)$ & $24.14-25.07$ & 0.92 \\
Presentation and accessibility of research $(6-30)$ & $6-37$ & $18.19(4.87)$ & $17.83-18.54$ & 0.90 \\
Quality of research $(6-30)$ & $6-30$ & $17.60(4.87)$ & $17.25-17.95$ & 0.92 \\
\hline
\end{tabular}

${ }^{\mathrm{a}}$ The possible range of scores is denoted in parentheses.

BARRIERS: Barriers to Research Utilization scale. 
Table 3. The 10 Top- and Bottom-Ranked Items of BARRIERS Scale with Means and Standard Deviations

\begin{tabular}{|c|c|l|l|}
\hline Rank Order & Subscale & & Mean (SD) \\
\hline \hline 1 & $\mathrm{~S}$ & There is insufficient time on the job to implement new ideas & $4.10(1.20)$ \\
\hline 2 & $\mathrm{~N}$ & The nurse does not see the value of research for practice & $3.98(1.18)$ \\
\hline 3 & $\mathrm{~S}$ & The facilities are inadequate for implementation & $3.95(1.14)$ \\
\hline 4 & $\mathrm{~S}$ & The nurse does not have time to read research & $3.88(1.14)$ \\
\hline 5 & $\mathrm{~N}$ & The nurse is unaware of the research & $3.77(1.12)$ \\
\hline 6 & $\mathrm{~S}$ & The nurse does not feel she has enough authority to change patient care procedures & $3.74(1.10)$ \\
\hline 7 & $\mathrm{~S}$ & The nurse feels that the results are not generalizable to his/her own setting & $3.61(1.11)$ \\
\hline 8 & $\mathrm{P}$ & Implications for practice are not made clear & $3.50(1.10)$ \\
\hline 9 & $\mathrm{~S}$ & Administration will not allow implementation & $3.45(1.20)$ \\
\hline 10 & $\mathrm{~N}$ & There is not a documented need to change practice & $3.34(1.17)$ \\
\hline 25 & $\mathrm{~N}$ & Research /articles are not published fast enough & $2.93(1.13)$ \\
\hline 26 & $\mathrm{R}$ & The amount of research information is overwhelming & $2.92(1.19)$ \\
\hline 27 & $\mathrm{R}$ & The literature reports conflicting results & $2.91(1.08)$ \\
\hline 28 & $\mathrm{~N}$ & The nurse is unwilling to change or try new ideas & $2.89(1.07)$ \\
\hline 29 & $\mathrm{~N}$ & The nurse see little benefit for him/herself & $2.81(1.03)$ \\
\hline
\end{tabular}

S: Organisational Characteristics, N: Nurse Characteristics, P: Presentation and Accessibility of Research, R: Quality of Research.

implementation' (mean=3.87, SD=1.16). The Cronbach's alpha coefficient of the FRU scale was 0.90 in this study.

\section{Relationships Between Nurses' Characteristics, Involve- ment in Research and Barriers to Research Utilization}

The results of the correlation tests between the nurses' mean scores of BARRIERS and FRU scales, their sociodemographic and clinical characteristics are summarized in Table 5. The correlations between the nurses' overall level of perceived barriers to research utilization and their mean age (Pearson's correlation $\mathrm{r}=0.18, \mathrm{p}=0.05$ ), year range of postregistration nursing experience (Spearman's rank correlation $\left.\mathrm{r}_{\mathrm{s}}=0.17, \mathrm{p}=0.05\right)$ and education level $\left(\mathrm{r}_{\mathrm{s}}=-0.20, \mathrm{p}=0.04\right)$ were statistically significant but low. Completion of basic research courses $(0=$ No; $1=$ Yes $)$ was moderately and significantly correlated with the nurses' involvement in clinical research (Point bi-serial correlation $\mathrm{r}_{\mathrm{pb}}=0.34, \mathrm{p}=0.01$ ) and their perceived barriers $\left(r_{p b}=-0.29, p=0.03\right)$. Therefore, the nurses without basic research skills training, a lower level of academic or professional qualification and longer postregistration experience were more likely to perceive a higher level of barriers to research utilization in their practices. In addition, the nurses who undertook research courses indicated more involvement in research than those who did not.

\section{Relationships between Nurses' Characteristics and Perceived Facilitators of Research Utilization}

As indicated in Table $\mathbf{5}$, the correlations between the nurses' perceived facilitators of research utilization and their education level $\left(\mathrm{r}_{\mathrm{s}}=0.31, \mathrm{p}=0.02\right)$, and perceived barriers to research utilization (Pearson's correlation $\mathrm{r}=-0.28, \mathrm{p}=0.01$ ), were statistically significant and negative. Otherwise, there were no significant correlations between the nurses' perceived facilitators and other socio-demographic variables.

\section{Comparison of Nurses' Perceived Barriers and Facilitators Between Selected Socio-Demographic Variables}

Results of one-way ANOVA test indicated that there was a significant difference between the mean score of nurses' perceived facilitators of research utilization and their education level $[\mathrm{F}(3,741)=2.762, \mathrm{p}=0.04]$. There were also significant differences between the mean score of the nurses' perceived barriers and their year range of nursing experience $[\mathrm{F}(4,740)=3.367, \mathrm{p}=0.01]$, as well as their education levels $[\mathrm{F}(3,741)=2.886, \mathrm{p}=0.04]$. Post-hoc analyses using the

Table 4. Item Means and Standard Deviations of FRU Scale $(\mathbf{N}=\mathbf{7 4 3})$

\begin{tabular}{|c|l|c|}
\hline Rank Order & \multicolumn{1}{|c|}{ Item } & Mean (SD) \\
\hline \hline 1 & Advanced education to increase your research knowledge base & $3.92(1.14)$ \\
\hline 2 & Improving availability and accessibility of research reports & $3.89(1.09)$ \\
\hline 3 & Enhancing managerial support and encouragement of research implementation & $3.87(1.16)$ \\
\hline 4 & Improving the understandability of research reports & $3.86(1.11)$ \\
\hline 5 & Employing nurses with research skills to serve as role models & $3.83(1.19)$ \\
\hline 6 & Conducting more clinically focused and relevant research & $3.79(1.13)$ \\
\hline 7 & Increasing the time available for reviewing and implementing research findings & $3.54(1.20)$ \\
\hline 8 & Providing colleague support networks/mechanisms & $3.52(1.25)$ \\
\hline
\end{tabular}


Table 5. Results of Correlation Tests of Nurses' BARRIERS and FRU Mean Scores and Selected Characteristics

\begin{tabular}{|c|c|c|c|c|c|c|c|}
\hline Variable & 1 & 2 & 3 & 4 & 5 & 6 & 7 \\
\hline 1. Mean age & 1.00 & $0.18^{\mathrm{a}}$ & 0.16 & 0.12 & 0.11 & $0.18^{\mathrm{a}}$ & $0.12^{\mathrm{a}}$ \\
\hline 2. Education level & & 1.00 & 0.11 & 0.13 & 0.10 & $-0.20^{\mathrm{a}}$ & $0.31^{\mathrm{a}}$ \\
\hline 3. Year range of post-registration experience & & & 1.00 & -0.11 & -0.10 & $0.17^{\mathrm{a}}$ & 0.10 \\
\hline 4. Nursing research course undertaken\# & & & & 1.00 & $0.34^{\mathrm{b}}$ & $-0.29^{a}$ & 0.14 \\
\hline 5. Participation in research\# & & & & & 1.00 & -0.12 & 0.13 \\
\hline 6. BARRIERS & & & & & & 1.00 & $-0.28^{b}$ \\
\hline 7. FRU & & & & & & & 1.00 \\
\hline
\end{tabular}

${ }^{\mathrm{a}}$ Significant correlation between two variables is denoted, with $\mathrm{p} \leq 0.05$.

${ }^{\mathrm{b}}$ Significant correlation between two variables is denoted, with $\mathrm{p} \leq 0.01$.

"Point bi-serial correlation test was used to test its relationship with the BARRIERS and FRU mean scores.

BARRIERS: Barriers to Research Utilization scale; FRU: Facilitators of Research Utilization scale.

Bonferroni multiple comparison procedures revealed that nurses with one to five years of nursing experience (mean= 78.64, $\mathrm{SD}=10.14$ ) perceived a significantly higher degree of barriers than those with 11 to 12 years of experience $($ mean= 82.30, $\mathrm{SD}=8.43$ ), with $\mathrm{t}=3.01, \mathrm{df}=741$ and $\mathrm{p}=0.05$. When compared with those with master's degrees or above, Chinese nurses with a diploma level of nursing education reported a lower extent of perceived facilitators (mean= 27.52, $\mathrm{SD}=8.57$ versus mean $=33.10, \mathrm{SD}=5.05$ ), with $\mathrm{t}=$ $3.54, \mathrm{df}=741$ and $\mathrm{p}=0.03)$, and a higher level of perceived barriers $($ mean $=85.72, \mathrm{SD}=9.83$ vs mean $=80.21, \mathrm{SD}=$ 11.02), with $\mathrm{t}=3.28, \mathrm{df}=741$ and $\mathrm{p}=0.04)$.

\section{DISCUSSION}

This was the first study conducted in mainland China to examine general nurses' perceived barriers to and facilitators of research utilization in nursing practice and their training and involvement in research. With a high level of support from the nurse managers in the delivery of the study questionnaires, an exceptionally high response rate $(92.8 \%)$ was achieved in this study. In previous similar studies, it was reported to be around $40 \%$ only (e.g., Hutchinson \& Johnston [4], Chau et al. [18]). It is interesting to note that about half of the nurses (i.e., 370 out of 743) in the four regional general hospitals did not receive any basic research course or in-service training in clinical research in the past three years and only $15.6 \%$ of them (i.e., 116 out of 743) were involved in clinical research. However, those who had recently received research training were found to be more involved in research activities, and also had fewer or lower degree of perceived barriers to research utilization in their practices. These findings highlight the importance of training in nursing research, either from basic training or continuous nursing education, to equip nursing students or staff with essential research knowledge and skills to critically appraise and participate in clinical research.

Although many similarities were found, the findings of this study identified a few major differences in the perceived barriers to and facilitators of research utilization from those reported in Western countries, as well as in Hong Kong Chinese nurses. These differences may improve our understanding of this topic and thus are discussed below.

\section{Nurses' Perceived Barriers to Research Utilization in China}

A majority of the Chinese nurses in this study perceived a higher degree of barriers when compared with registered nurses in other countries. The Chinese nurses rated $69 \%$ of the 29 'barriers' items as moderate or great barriers, which was comparable with $65 \%$ in both the U.S. [24] and U.K. nurses [34]. Nevertheless, this was much higher than nurses in Australia [4] and Hong Kong [6], who rated about $40 \%$ and $41 \%$ of them respectively as moderate or great barriers. Therefore, the Chinese nurses in this study reported a higher mean total scale (mean $=89.3$ and $\mathrm{SD}=19.6$ ) and subscale (from mean=17.6 and $\mathrm{SD}=4.9$ to mean=26.1 and $\mathrm{SD}=6.2$ ) scores on the BARRIERS scale, when compared with Western nurses. Such great differences in their perceived barriers to research utilization may be explained by the much lower level of nursing education, traditional education curricula (e.g., the lack of research courses), inadequate support for nursing research, and lack of policy and guidelines on evidence-based practice found in China.

In this study, the greatest perceived barriers to research utilization among the Chinese nurses were related to organizational or setting factors. They mainly included inadequate time to implement new ideas for practice learned from research evidence, lack of facilities to review or conduct research, the feeling of a lack of authority to change practice, and the scarcity of research findings generalizable to their settings [35]. These findings were also found significant in the large-scale survey studies among nurses in different countries and practice settings. For instance, these four barriers were identified as significant among U.K. nurses in both hospital and community care settings [17], Swedish nurses within regional general hospitals [36], Canadian pediatric nurses [36], and most recently, among Hong Kong general nurses in public hospitals [18]. Similar to general nurses in Turkey [8], the Chinese nurses in this study indicated that they considered shortage of manpower, inadequate facilities, and lack of authority or support from the health care team to change practice as the major barriers to research utilization in their practice settings.

In addition, two of the top five barriers were 'lack of time to implement new ideas' and 'lack of time to read research reports' under the subscale 'Organizational Characteristics'; 
these barriers were also rated highly in other studies (e.g., Hutchinson \& Johnston [4], Mehrdad, et al. [5]). As in Turkey and many Asian countries [6,8], there is a shortage of nursing manpower and nurses are practicing under an overwhelming workload. Therefore, time constraints for literature review, applying research evidence into practice and participation in research activities has become a major barrier to their evidence-based practice. Although a set of broad, countrywide evidence-based health care policies has been stipulated by the Ministry of Health in mainland China [32,33], there are no specific policies and guidelines in individual hospitals and other health care organizations for the implementation of nursing research or evidence-based practice. For this reason, together with the insufficient manpower, high workload and time constraints, Chinese nurses may perceive there to be many inhibitors of research utilization or evidence-based practice, such as 'the facilities are inadequate for implementation', 'the results are not generalizable to our own setting' and 'the administration will not allow implementation', which were rated as three of the top 10 barriers by the nurses in this study. The utmost importance of organizational support for research utilization should be reinforced by establishing innovative strategies and sufficient resources and facilities for research implementation such as setting up centers for research and evidence-based practice, clearly defined guidelines for research implementation and utilization in clinical areas and more opportunities for nursing research training [18,37]. These supportive strategies can in turn nurture research appraisal and a culture for research utilization in different clinical areas [10].

The sixth top-ranked barrier related to the organizational factor, 'they do not feel they have enough authority to change patient care procedures' echoes the findings of Parahoo's [23] study. Parahoo [23] suggested that registered nurses perceived research findings as difficult to implement in settings where they were not recognized as independent professionals and autonomous members of the health care team, and thus nursing research was not supported. This finding is also consistent with the idea of the fifth barrier 'the nurse feels that the results are not generalizable to his/her own setting', confirming that nurses find it hard to implement research evidence in practice when they perceive themselves to be lack of the authority and autonomy to make changes in their practice. Nevertheless, Chinese nurses may recognize their current low professional status in the health care system. They need managerial support to increase their professional autonomy in clinical decision-making and collaborative practice and enhance their knowledge and skills in research in order to implement evidence-based nursing practice.

In contrast with most recent studies (e.g., Chau et al. [18] in Hong Kong, Hutchinson and Johnston [4] in Australia and Mehrdad et al. [5] in Iran), the Chinese nurses in this study did not perceive that "physicians will not cooperate with implementation' or 'other staff are not supportive of implementation' were important barriers to research utilization. This may reveal that the relationships between nurse and physician or nurse and members of the health care team in China are satisfactory and thus they are considered to collaborate with each other satisfactorily in the implementation of research evidence into practice.
Therefore, it is important for physicians and other members of the health care team to acknowledge that research is also a nursing responsibility. In addition, the implementation of research in nursing practice is also essential to ensure the high quality of patient care and to optimize benefits to all parties in the health care system.

Another interesting finding of this study is that two items, 'the nurse does not see the value of research for practice' and 'the nurse is unaware of the research', were rated as the second and fifth top-ranked barriers to research utilization, respectively. However, these items have often been rated among the least important barriers in most countries (i.e., in the bottom 10 positions) [38]. This may be related to the comparatively lower education level of Chinese nurses compared with those in Western and other developed Asian countries. This may also be because very few of them had recently undertaken research training or participated in research activities. Recent studies have suggested that nurses who have neither undergone research training nor been assigned with responsibility and authority concerning research activities are unable to critically appraise research evidence, unwilling to spend time accessing and reading research, and thus unaware of the significance of research to practice $[8,39]$. To investigate their recognition of the importance of nursing research and/or evidence-based practice and the reasons for their low awareness of its value to practice, in-depth qualitative research is recommended in nurses with different levels of education and research experience.

It is also interesting to note that older and senior Chinese nurses (with more years of clinical experience) perceived more barriers to research utilization than younger and more junior ones. This significant result has not yet been found in other studies. It may be explained by two possible reasons that are specific to Chinese nurses. First, the senior nurses were trained using a traditional, hospital-based education curriculum during the 1980s and 1990s, when nurses graduated with limited knowledge and skills in nursing research, statistics and evidence-based practice. Second, many younger nurses have undertaken nursing research courses in their undergraduate or postgraduate education, or have been nominated by hospital managers to attend inservice training courses on clinical research or evidencebased practice. They are also expected by managers or administrators to be more likely or responsible for being involved in research or to apply research findings to clinical practice [8,14]. Being taught about research and being expected to use research in practice may be important factors contributing to higher sensitivity and more positive attitudes and values towards research, thus perceiving lower barriers to research utilization. These important factors and their relationships are worthy of further exploration in future research.

\section{Chinese Nurses' Perceived Facilitators of Research Utilization}

For the perceived facilitators, the Chinese nurses indicated a moderate level of facilitators of research utilization in their settings, with the eight items' mean scores ranging from $3.52(\mathrm{SD}=1.25)$ to $3.92(\mathrm{SD}=1.14)$. These results were lower than that of the Australian nurses in 
Hutchinson and Johnston's [4] study, which reported the item means of the FRU scale as being from $4.04(\mathrm{SD}=1.22)$ to 4.52 ( $\mathrm{SD}=0.93)$. The three top-ranked facilitators are also very different between the two studies. While advanced education, the availability and accessibility of research reports and managerial support and encouragement of research implementation were considered the greatest facilitators among the Chinese nurses in this study, the Australian nurses indicated that increasing time for reviewing and implementing research findings, conducting more clinically focused and relevant research and providing colleague support mechanisms were their most important facilitators of research utilization. This finding may again reflect a high need for research education and the provision of more resources and organizational support and encouragement to enable Chinese nurses to initiate or implement evidence-based practice. This understanding also echoes the findings on the perceived barriers in the previous section regarding Chinese nurses' lack of research knowledge, inadequate organizational support for research, and lack of awareness of research evidence and its values in patient care.

In addition, a significant positive correlation between nurses' perceived facilitators and their education level was also found in this study, similar to the findings of another recent study by Chau et al. [18] of Hong Kong Chinese nurses. Most Australian nurses (about 89\%) who received tertiary nursing education might perceive more organizational and health care team support and facilitation of research utilization than Chinese nurses, of whom fewer than half $(43 \%)$ have undergraduate nursing qualifications. As a result, the Chinese nurses rated 'advanced education to increase your research knowledge base' and 'improving the availability and accessibility of research reports' as the most important facilitators of research utilization. Nevertheless, the Hong Kong Chinese nurses in Chau et al.'s [18] study rated 'enhancing managerial support and encouragement of research implementation' and 'providing colleague support networks or mechanisms' as the most important facilitators, which are quite similar to the options of Australian nurses $[4,15]$. This comparison indicates a significant difference across countries in terms of facilitators of research utilization. It also reveals that the successful dissemination and implementation of research evidence in nursing practice is contingent upon an understanding of both potential barriers and facilitators in terms of its specific organizational context and culture, as well as the nursing and other health care staff $[18,23]$. In mainland China, nurse managers need to provide the time and resources necessary for advanced research education and implementation of research; and nurse educators should include different levels of research courses such as basic and advanced research methods and statistical analysis into continuing nursing education programs $[29,32]$.

Similar to other nurses in developing countries, many Chinese nurses lack research knowledge and skills, and have limited resources and support for research utilization; some also find it difficult to critically appraise research evidence [32]. Indeed, Chinese nurses are unable to access the online databases of healthcare journals or other research evidences in their workplaces, or hospital libraries. They can only make a request for a research article on an individual basis for consideration, thus experiencing great difficulties in research-based practice. Therefore, they would place relatively less value on research utilization and have inadequate opportunities for research involvement. Recently, there have been a few initiatives emphasizing the importance of evidence-based nursing practice in different cities and provinces. However, full support for evidence-based practice should be provided by the central government or Ministry of Health, through which more Chinese nurses can be better equipped with research knowledge and skills and increased responsibility and opportunities for being involved in the research implementation and utilization. In this way, Chinese nurses could change their attitudes and values towards research utilization for improving their practices, thus providing high quality patient care.

Similar to other developing countries, clinical nurses in mainland China have been encountering with high levels and great varieties of barriers to research utilization. With the findings of this study, a few strategies in promoting research utilization in developing countries are suggested. First, in lack of policy and guidelines on evidence-based practice in hospitals and/or the whole country, it is of utmost importance to set up country- or hospital-wide healthcare or nursing policies by the ministry of health, or the respective health authorities, for the implementation of nursing research or evidence-based practice. To achieve this guidelines or policy, adequate organizational support for research appraisal and utilization should be reinforced by establishing innovative strategies and sufficient resources and facilities for research implementation such as setting up centers for research and evidence-based practice and clearly defined guidelines for research implementation and utilization in clinical areas. Research-based practice can also be set as an expectation of 'usual' nursing practice, and rewards can be offered to those who have demonstrated successful research implementation and utilization in their practices [37].

Second, sufficient preparation of nurses for research appraisal and implementation and evidence-based practice should be recommended. Nursing research courses, whether at basic or advanced level, should be an integral component of the education curriculum of different levels of university and in-service nursing education programs in order to better facilitate the implementation of research- or evidence-based practice in different clinical settings.

Third, with better preparation of nurses for research knowledge and skills, there is a need for recognition of their competence in research utilization from their working hospitals or organizations. They should be given sufficient authority and autonomy for making changes in their practices, with collaborative decisions in the healthcare team. As the result, nurses will see the value of research for practice and be more aware of high-quality research findings as best evidence in their daily practices.

Lastly, creating a supportive environment to facilitate access and utilization of research findings is an integral part of evidence-based nursing practice $[33,38]$, and thus should be considered by the health care institutions and authorities. Collaboration, cooperation and support amongst different levels of medical, nursing and other disciplines in nurturing cultures on evidence-based practice and developing research projects in clinical settings. 


\section{LIMITATIONS OF THE STUDY}

Several limitations of this study should be considered. First, even though there was a high response rate and reasonably large sample size, the convenience sampling of general nurses in four of over 60 regional hospitals in the two provinces, or of more than 300 in the whole country, may limit the representativeness of the findings and thus their generalization to the nursing population in mainland China. A territory-wide epidemiological study should be considered to investigate Chinese nurses' perceived barriers to and facilitators of research utilization and their correlated factors. Smaller hospitals or clinics with nursing specialties and advanced nursing practice such as cardio-thoracic, maternal, psychiatric, or palliative care in various hospitals or districts should also have been included in order to understand the perceived barriers to and facilitators of research utilization among nurse specialists and compare their perceptions between specialty groups and different geographical regions in China, and across countries.

Second, the use of a self-reported questionnaire, which is considered the most convenient and economical data collection method, rendered it difficult to assess or avoid response biases due to misunderstanding of the questionnaire items, or to the participants responding according to what they perceived to be socially desirable. In addition, some of the researchers are the nurse administrators of the hospitals under study, thus their participation in the data collection and/or data analyses might have enhanced the nurses' response rate and/or tendency to give desirable responses, thus affecting the interpretation of the results.

Third, the conclusion of this study is tentative because of its cross-sectional design, the fact that it was not hypothesisdriven, and due to the use of a Chinese version of the BARRIERS and FRU scales with acceptable reliability and validity in small-sized convenience samples of Chinese nurses [6,18]. In addition, only a few socio-demographic variables were selected to test the correlation and subgroup differences in this study. More appropriate and comprehensively selected potential factors that may affect research utilization based on the previous research findings in both Chinese and Western nursing populations should be included in future research, for example, using the hypothesized model testing approach. Further testing of the psychometric properties of the two Chinese versions of the research instruments is recommended to ensure accurate and valid measurement of the potential barriers to and facilitators of research utilization.

\section{CONCLUSIONS}

This study identified Chinese nurses' perceived barriers to and facilitators of research utilization in mainland China. Overall, the Chinese nurses perceived a higher degree and a wider diversity of barriers to research utilization in their practice, when compared with their counterparts in Western and other Asian countries. Among them, inadequate organizational support and limited knowledge and skills in research implementation and utilization were the two topranked barriers perceived by the nurses, while the need for advanced education and resource support were rated as the most important facilitators. Administrators' and managers' creation of a positive and supportive organizational environment is therefore of utmost importance in enhancing research implementation and utilization. The sociodemographic factors of the Chinese nurses, including their education level, age, years of post-registration nursing experiences, and research training, were found to be correlated with their perceived barriers to and facilitators of research utilization in practice. These barriers and facilitators, together with more potential socio-demographic and organizational correlates, should be further examined and carefully addressed to provide a high quality of evidenced-based nursing practice in rapidly developing countries such as mainland China.

\section{CONFLICT OF INTEREST}

The authors confirm that this article content has no conflict of interest.

\section{ACKNOWLEDGEMENTS}

The authors would like to express their gratitude to the nurses who participated in this study and completed the questionnaire. The authors also thank the hospitals under study and their staff for providing assistance and support in data collection.

The study was supported by the Departmental Research Fund, School of Nursing, The Hong Kong Polytechnic University, Hong Kong SAR, PR China.

\section{REFERENCES}

[1] Niederhauser V, Kohr L. Research endeavors among pediatric nurse practitioners (REAP) study. J Pediatr Health Care 2005; 19: $80-9$.

[2] Hommelstad J, Ruland CM. Norwegian nurses' perceived barriers and facilitators to research use. ARON J 2004; 79: 621-34.

[3] McSherry R, Simmons M, Abbott P. Evidence-informed nursing: A guide for clinical nurses. London, UK: Routledge 2002; pp. 40-60.

[4] Hutchinson AM, Johnston I. Bridging the divide: a survey of nurses' opinions regarding barriers to, and facilitators of, research utilization in the practice setting. J Clin Nurs 2004; 13: 304-15.

[5] Mehrdad N, Salsali M, Kazemnejad A. The spectrum of barriers to and facilitators of research utilization in Iranian nursing. J Clin Nurs 2008; 17: 2194-202.

[6] Chien WT. A survey of nurses' perceived barriers to research utilization in Hong Kong. J Clin Nurs 2010; 19(23-24): 3584-6.

[7] Salsali M, Mehrda N. Iranian nurses' constraint for research utilization. BMC Nurs 2009; 8: 9.

[8] Uysal A, Temel AB, Ardahan M, Özkahraman Ş. Barriers to research utilization among nurses in Turkey. J Clin Nurs 2010; 19(23-24): 3443-52.

[9] Eller SL, Kleber E, Wang SL. Research knowledge, attitudes and practices of health professionals. Nurs Outlook 2003; 51: 165-70.

[10] Retsas A. Barriers to using research evidence in nursing practice. $\mathbf{J}$ Adv Nurs 2000; 31: 599-606.

[11] Valizadeh L, Zamanzadeh V. Research utilization and research attitudes among nurses working in teaching hospitals in Tabriz, Iran. J Clin Nurs 2003; 12: 928-30.

[12] Estabrooks CA, Dersken L, Winther C, et al. The intellectual structure and substance of the knowledge utilization field: A longitudinal author co-citation analysis, 1945 to 2004. Implement Sci 2008; 3: 1-22.

[13] Squires JE, Estabrooks CA, Gustavsson P, Wallin L. Individual determinants of research utilization by nurse: A systematic review update. Implement Sci 2011; 6: 1.

[14] Rodgers SE. A study of the utilization of research in practice and the influence of education. Int J Nurs Stud 2000; 36: 335-43.

[15] Hutchinson AM, Johnston L. Beyond BARRIERS scale: commonly reported barriers to research use. J Nurs Adm 2006; 36: 189-99.

[16] Meijers JMM, Janssen MAP, Cummings GG, Wallin L, Estabrooks CA, Haltens RYG. Assessing the relaitonships between contextual 
factors and research utilization in nursing: Systematic literature review. J Adv Nurs 2006; 55: 622-35.

[17] Bryar R, Closs S, Baum G, et al. The Yorkshire BARRIERS project: Diagnostic analysis of barriers to research utilization. Int J Nurs Stud 2003; 40: 73-8

[18] Chau J, Lopez V, Thompson D. A survey of Hong Kong nurses' perceptions of barriers to and facilitators of research utilization. Res Nurs Health 2008; 31: 640-9.

[19] LaPierre E, Ritchey K, Newhouse R. Barriers to research use in the PACU. J Perianesth Nurs 2004; 19: 78-83.

[20] McKenna H, Ashton S, Keeney S. Barriers to evidence-based practice in primary care. J Adv Nurs 2004; 45: 178-89.

[21] Thompson C, McCaughan D, Cullum N, Sheldon T, Raynor P. Barriers to evidence-based practice in primary care nursing - why viewing decision-making as context is helpful. J Adv Nurs 2005; 52: 432-44.

[22] Oranta O, Routasalo P, Hupli M. Barriers to and facilitators of research utilization among Finnish Registered nurses. J Clin Nurs 2002; 11: 205-13.

[23] Parahoo K. Barriers to, and facilitators of, research utilization among nurses in Northern Ireland. J Adv Nurs 2000; 31: 89-98.

[24] Funk SG, Champange MT, Wiese RA, Tornquist EM. BARRIERS: the barriers to research utilization scale. Appl Nurs Res 1991; 41: $39-45$.

[25] Rogers EM. Diffusion of innovations. $4^{\text {th }}$ ed. New York: The Free Press 1995.

[26] Patiraki E, Karlou C, Papadopoulou D, et al. Barriers to implementing research findings in cancer care: the Greek registered nurses' perceptions. Eur Oncol Nurs Soc 2004; 8: 245-65.

[27] Sitzia J. Barriers to research utilization: the clinical setting and nurses themselves. Eur J Oncol Nurs 2001; 5: 154-64.
[28] Leung G, Bacon-Shone J. Hong Kong's health system: Reflections, perspectives and visions. Hong Kong: Hong Kong University Press 2006.

[29] Wang Y, Chien WT, Twinn S. An exploratory study on baccalaureate-prepared nurses' perceptions regarding clinical decision-making in mainland China. J Clin Nurs 2012; 21: 170615.

[30] Portney LG, Watkins MP. Foundation of Clinical Research: Application to Practice. $2^{\text {nd }}$ ed. Philadelphia, NJ: Prentice Hall 2000

[31] Wang MQ, Fitzhugh E, Westerfield RC. Determining sample size for simple-random surveys. Health Values 1995; 19(3): 53-6.

[32] Eddins EE, Hu J, Liu H. Baccalaureate nursing education in China: issues and challenges. Nurs Educ Perspect 2011; 32: 30-3

[33] Lu H, While AE, Barriball KL. Role perceptions and reported actual role content of hospital nurses in mainland China. J Clin Nurs 2008; 17: 1011-22.

[34] Pallen N, Timmins F. Research-based practice: Myths or reality? A review of the barriers affecting research utilization in practice. Nurs Educ Pract 2002; 2: 99-108.

[35] Kajermo KN, Undén M, Gardulf A, et al. Predictors of nurses' perceptions of barriers to research utilization. J Nurs Manag 2008 16: 305-14.

[36] McCleary L, Brown GT. Barriers to paediatric nurses' research utilization. J Adv Nurs 2003; 42: 364-72.

[37] Maljanian R, Caramanica L, Taylor SK, MacRae JB, Bland DK. Evidence-based nursing practice, Part 2: Building skills through research roundtables. J Nurs Adm 2002; 32: 85-90.

[38] Funk SG, Tornquist EM, Champange MT. Barriers and facilitators of research utilization. Nurs Clin North Am 1995; 30: 395-407.

[39] Temel AB, Uysal A, Ardahan M, Özkahraman Ş. Barriers to Research Utilization scale: psychometric properties of the Turkish version. J Adv Nurs 2010; 66: 456-64.

(C) Chien et al.; Licensee Bentham Open.

This is an open access article licensed under the terms of the Creative Commons Attribution Non-Commercial License (http://creativecommons.org/licenses/by-nc/3.0/) which permits unrestricted, non-commercial use, distribution and reproduction in any medium, provided the work is properly cited. 